\title{
Sonographic Evaluation of Incidental Synchronous Masses in Patients with Breast Cancer: Clinical Significance and Diagnostic Workup
}

\author{
(D) Sara Rehman ${ }^{1}$, (1) Imran Khalid Niazi ${ }^{1}$, (1) Muhammad Atif Naveed ${ }^{1}$, (1) Ainy Javaid ${ }^{1}$, (1) Bushra Rehman ${ }^{2}$ \\ ${ }^{1}$ Department of Radiology, Shaukat Khanum Memorial Cancer Hospital and Research Centre, Lahore, Pakistan \\ ${ }^{2}$ Department of Breast Surgery, Shaukat Khanum Memorial Cancer Hospital and Research Centre, Lahore, Pakistan
}

\begin{abstract}
Objective: This study aimed to evaluate the diagnostic accuracy of breast ultrasonography in classifying incidental satellite masses as benign or malignant in patients with breast cancer and to assess its effect on their surgical management.

Materials and Methods: Ultrasound-guided fine needle aspiration/biopsy was performed in 288 satellite masses of 225 patients with breast cancer. Two radiologists retrospectively reviewed the sonograms of these masses and classified them as benign or malignant and compared this feature with the results of the histopathological examination. The location of the satellite mass and type of surgery were also documented.

Results: Of the 288 satellite masses, 139 were located in the same quadrant, 95 in different quadrants, while 54 were in the contralateral breast. Of the 123 sonographically benign masses, 106 showed benign pathological outcome, and from 165 sonographically malignant masses, 127 were found malignant on histopathology/cytology. McNemar's chi-square was 7.27 ( $\mathrm{p}$-value=0.007), showing statistically significant association between sonographic features and pathological outcome of satellite masses. The sensitivity, specificity, positive and negative predictive values, and accuracy were $88.2 \%, 73.6 \%, 77 \%$, $86.1 \%$, and $80.9 \%$ respectively. Based on these findings, 61 patients underwent lumpectomy limited to a single tumor, 52 underwent extended resection, 78 underwent mastectomy, four underwent lumpectomy for the contralateral breast, and bilateral mastectomies were performed in another four patients. Surgery was not performed in 26 patients.
\end{abstract}

Conclusion: Although ultrasound is an effective tool for the detection and characterization of incidental satellite masses in patients with breast cancer, biopsy is imperative to ascertain the pathological diagnosis and, therefore, select the most appropriate surgical plan.

Keywords: Breast cancer, multicentric, multifocal, satellite, ultrasonography

Cite this article as: Rehman S, Niazi IK, Naveed MA, Javaid A, Rehman B. Sonographic Evaluation of Incidental Synchronous Masses in Patients with Breast Cancer: Clinical Significance and Diagnostic Workup. Eur J Breast Health 2021; 17(1): 28-35.

\section{Introduction}

Multifocal-multicentric breast cancers (MMBC) are defined as two or more discrete synchronous tumors in the same breast (1). Breast ultrasonography (USG) is essential for the diagnosis and management of patients with breast cancer. Although breast cancer has characteristic imaging features, various benign diseases of the breast can also have similar appearance; therefore, if incidental findings are identified during diagnostic workup of a patient presenting with a breast lump, preoperative biopsies must be performed to characterize the lesion and to direct management (2). Familiarity with benign breast diseases increases the radiologists' confidence after a biopsy, allays patient fears, and avoids unnecessary surgical excision. MMBC had been traditionally treated aggressively with mastectomy until the publication of randomized trials of quadrantectomy and radiotherapy. Breast conservative treatment (BCT) is a reasonable option for selected patients with MMBC (3-5). BCT requires a thorough preoperative radiological workup, assessment of risk factors, and multidisciplinary team (MDT) discussion.

This study was carried out to identify the diagnostic accuracy of breast USG in classifying incidental synchronous breast masses as benign or malignant in patients with biopsy-proven breast cancer, to identify correlation with histopathology/cytology findings of ultrasound (US)-guided biopsy/fine needle aspiration (FNA), and to assess the effect of these findings on the surgical management of these patients.

\section{Materials and Methods}

\section{Study design and patient selection}

Our hospital institutional review board approved this retrospective data collection and analysis and waived the need for informed consent from all patients (EX-15-02-19-01). The Hospital Information System was searched for records of patients with breast cancer from Jan 1, 2017, 
to December 31, 2018. A total of 697 patients with breast cancer presented to our hospital within this time period. These patients underwent bilateral breast mammography and USG, followed by USguided FNA/biopsy. The primary presenting mass was subjected to US-guided biopsy using a 14-gauge Magnum needle (Bard, Murray Hill, NJ, USA), obtaining at least 3-5 cores. For the satellite masses, either US-guided biopsy using the aforementioned technique or USguided FNA was performed using a 25-gauge hypodermic needle. Onsite evaluation was done by cytotechnologists who assessed the sample for adequacy. Satellite masses with definite benign features [Breast Imaging Reporting and Data System (BI-RADS)] such as fibroadenoma with benign calcifications or hyperechoic lipomas were not subjected to US-guided FNA/biopsy (6). Similarly, in patients with extensive multifocal/multicentric (MF/MC) disease, regional microcalcifications on mammograms, or inflammatory carcinoma, unnecessary intervention was not employed, as it would not change the management. Satellite masses for which pathological diagnosis was not available were excluded from the study. We included 225 patients with breast cancer presenting with US-detected synchronous breast masses, which were occult on clinical examination and mammography.

Breast USG is usually performed by a radiology fellow or a resident doctor in our clinical setting and reviewed by one of the six breast imaging consultants (with at least 4-10 years of experience). Linear array transducers of 7.5-10 MHz (Toshiba Aplio XG, Toshiba Xario 100, and Toshiba Aplio 500, Cannon Medical Systems, Japan) were used. We routinely review mammograms before performing USG, as well as US-guided fine needle aspiration cytology (FNAC) or core biopsy; hence, the radiologist performing the procedure had an idea about mammographic findings.

The following data were obtained from the Hospital Information System: age, sex, laterality, primary diagnosis, number of satellite masses, location of satellite masses, distance from the main mass, US features of each satellite mass, histopathology/cytology of satellite mass, and surgical management.

\section{Sonographic features}

Sonograms were reviewed by a radiology fellow and resident doctor under the supervision of two consultant radiologists with 5-10 years of experience. All satellite masses were evaluated for sonographic features regarding margins (circumscribed, spiculated, indistinct, or microlobulated), shape/orientation (oval, parallel, or not parallel), echogenicity (hyperechoic, isoechoic, or hypoechoic), and vascularity on color Doppler US (absent, increased, or not assessed). Presence of $>3$ satellite masses was considered a multiple occurrence. Masses with at least one malignant feature were classified as sonographically malignant (BI-RADS 4-5), whereas masses with all benign features are labeled as sonographically probably benign (BI-RADS 3) according to the American College of Radiology (ACR) BI-RADS Atlas $5^{\text {th }}$ Edition (Appendix 1) (6). According to the ACR guidelines, tissue diagnosis is recommended for the management of BI-RADS 4-5 masses, while short-interval follow-up is required for BI-RADS 3 lesions ( $>0$ but $\leq 2 \%$ likelihood of malignancy); however, considering the patient's concurrent malignancy, we performed biopsy/FNA for definitive diagnosis.

Clock location in the breast and distance from the main mass were documented and then classified as ipsilateral and contralateral, with further subclassification into the same quadrant or different quadrant, if the mass is located in the ipsilateral breast.
The histopathology/cytology of each satellite mass was also reviewed and categorized as benign or malignant. Type of surgery was tabulated in a Microsoft Excel sheet.

For simplification, in this study, we did not take into account the size of the satellite mass, as size is not an indicator of malignant risk. Moreover, surgical management considers patients with good postchemotherapy response on USG as a good candidate for BCT. In our hospital, magnetic resonance imaging of the breasts is not routinely performed due to limited resources. Post-surgical pathology was not included, as most of the patients undergo surgery post neoadjuvant chemotherapy and the histopathology of mastectomy/lumpectomy specimen may not show any residual tumor in cases with complete treatment response.

\section{Statistical analysis}

Data analysis was done using SPSS v25 (IBM Corp., Armonk, NY, USA). Epitools, an online software, was used to calculate the sensitivity, specificity, positive predictive value (PPV), negative predictive value (NPV), and McNemar test value. Graphs were generated using Microsoft Excel.

\section{Results}

From January 1, 2017, to December 31, 2018, a total of 697 patients with breast cancer presented to our hospital. In total, 225 patients had more than one mass detected by breast USG. All patients were female (mean age: 45.2 years; range: 23-77 years). Moreover, 141 patients had one satellite mass, 50 had two, 17 had three, and 17 had multiple satellite masses. US-guided FNA was performed for 53 masses, while biopsy was performed for 235 satellite masses. The final histopathology/cytology results for all 288 masses are described in Table 1, showing an equal number of benign and malignant entities. The location of satellite nodules in relation to the main mass and the incidence of benign or pathological outcome according to each category are shown in Table 2.

Pathological outcome according to the US feature of the margin of satellite masses is shown in Figure 1. In this study, 106 (73.6\%) benign masses had circumscribed margins, and one-third of the malignant masses showed indistinct margins (52 masses; 36\%). As regards shape and orientation, Figure 2 shows that most of the benign masses (126 masses $87.5 \%$ ) had parallel orientation; however, nearly the same number of malignant masses demonstrated parallel (61 masses; $42.4 \%$ ) and not parallel orientation (58 masses; 40.3\%). All malignant masses were hypoechoic (100\%), and $95 \%$ of benign masses (137 masses) were hypoechoic, while $4.9 \%$ (7 masses) were isoechoic. No

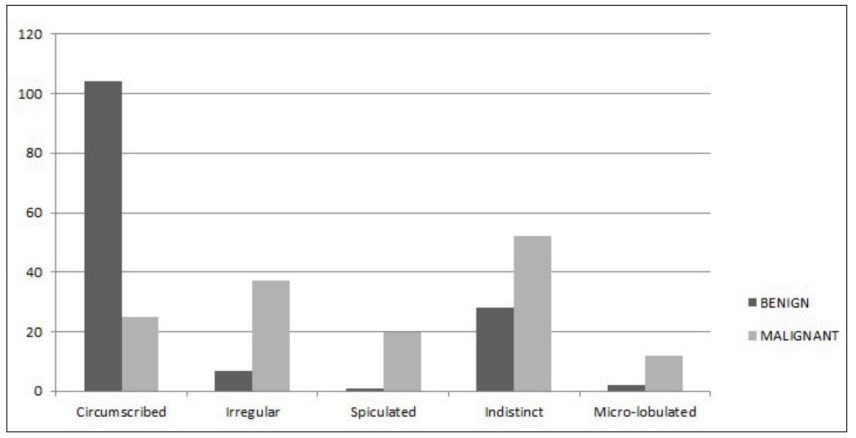

Figure 1. Pathological outcome of benign versus malignant masses according to the sonographic features of the margins of the satellite mass 
Table 1. Histopathology/cytology outcome of synchronous satellite masses after ultrasound-guided core biopsy/fine needle aspiration

\section{Histopathology/cytology outcome}

Benign

Fibroepithelial lesion/fibroadenoma

Breast tissue with fibrocystic change

Stromal fibrosis/adenosis/hyalinization

Sclerosing adenosis

Proliferative breast disease with/without atypia/antidiuretic hormone

Benign breast parenchyma/aspirate

Papillary lesion

Reactive intramammary lymph node

Fibrocollagenous/fibroadipose tissue/with fat necrosis

Chronic inflammation

Malignant

Mammary carcinoma (fine needle aspiration)

Ductal carcinoma in situ

Invasive ductal carcinoma

Invasive lobular carcinoma

Metastatic intramammary lymph node

Spindle cell carcinoma

Numbers in parentheses are percentages

Table 2. Location of synchronous satellite masses in relation to the main mass and incidence of benign and malignant masses according to location

\section{Location}

Ipsilateral breast

Same quadrant

Different quadrant

Contralateral breast

Numbers in parentheses are percentages

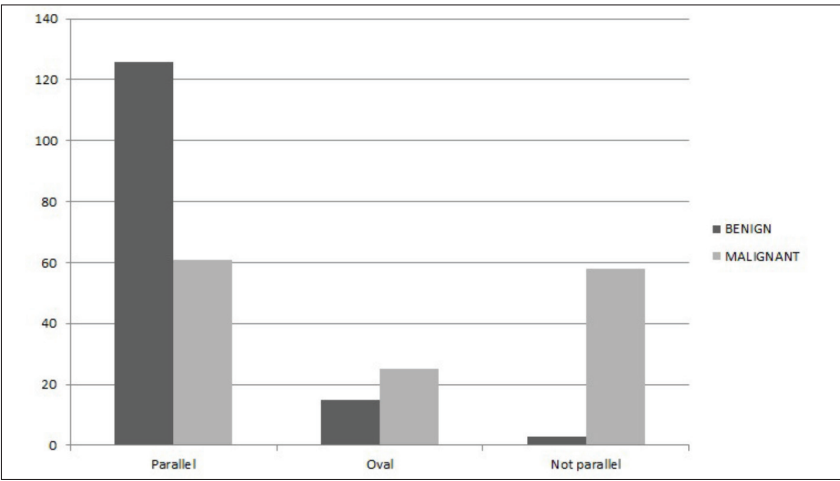

Figure 2. Pathological outcome of benign versus malignant masses according to the sonographic features of the shape/orientation of satellite masses

\section{Number of satellite masses}

$144(50)$

35

4

42

5

11

26

4

9

7

1

$144(50)$

33

14

91

2

hyperechoic mass was recorded in this study, as a hyperechoic mass with smooth margins and parallel orientation can be confidently labeled as lipoma (BI-RADS 2) and biopsy is not required. Eleven malignant masses showed increased vascularity, and 22 showed absent flow on color Doppler. Twenty-seven benign masses had no vascularity, while one had increased flow on color Doppler. Vascularity was not assessed in a significant number of satellite masses (227 masses; 78.8\%).

In this study, 123 masses were classified as sonographically probably benign (BI-RADS 3), 106 of these masses showed benign pathological outcome on histopathology/cytology, and 17 masses were malignant. Table 3 shows the relative incidence of malignant entities in this category. Of the 165 masses classified as sonographically malignant (BI-RADS 4-5), 127 were malignant on final the histopathology/

Table 3. Malignant pathological outcome of sonographically benign-appearing satellite masses

\section{Cytology/histopathology}

Mammary carcinoma

(fine needle aspiration)

Ductal carcinoma in situ

Invasive ductal carcinoma

Invasive lobular carcinoma

Metastatic intramammary lymph node

Spindle cell carcinoma
Number of satellite masses 
cytology, whereas 38 masses were benign. Table 4 shows the occurrence of different benign etiologies in sonographically malignant masses. A statistically significant association was found between sonographic features and pathological outcome of satellite masses (McNemar's chi-square, 7.27; $\mathrm{p}=0.007$ ). The sensitivity, specificity, PPV, NPV, and accuracy of sonographic classification of satellite masses into benign and malignant were $88.2 \%, 73.6 \%, 77 \%, 86.1 \%$, and $80.9 \%$ respectively.

The final surgical outcome is shown in Figure 3. Sixty-one patients had BCT with lumpectomy limited to a single tumor. In this group, all satellite masses were proven benign on histopathology/FNA, so unnecessary surgery was not performed. Patients with malignant satellite masses that required excision of the satellite mass along with the main mass in the same breast were grouped in the same category. This category included wire-guided excision of the satellite mass, wider excision of the main mass including the satellite mass, double lumpectomy for two masses, or quadrantectomy. Fifty-two patients were placed in this group, of which 51 had single malignant satellite mass (same quadrant, $n=37$; different quadrant, $n=14$ ), while one patient had two malignant satellite masses in the same quadrant. Seventy-eight patients underwent modified radical mastectomy/ mastectomy of one breast, and this included 39 patients with two or more malignant satellite masses, 16 patients with single malignant satellite mass in the same quadrant, 11 patients with single satellite mass in different quadrant, and 12 patients with benign satellite mass. BCT was not possible for these 12 patients with unifocal disease due to multiple factors, which included poor chemotherapy response, small breast size, patients' preference, or high-grade ductal carcinoma

Table 4. Benign pathological outcome of sonographically malignant appearing satellite masses

\section{Cytology/histopathology}

Fibroepithelial lesion/fibroadenoma

Stromal fibrosis/adenosis/

hyalinization

Sclerosing adenosis

Proliferative breast disease

Benign breast parenchyma

Fibrocollagenous/adipose tissue/fat necrosis

Chronic inflammation

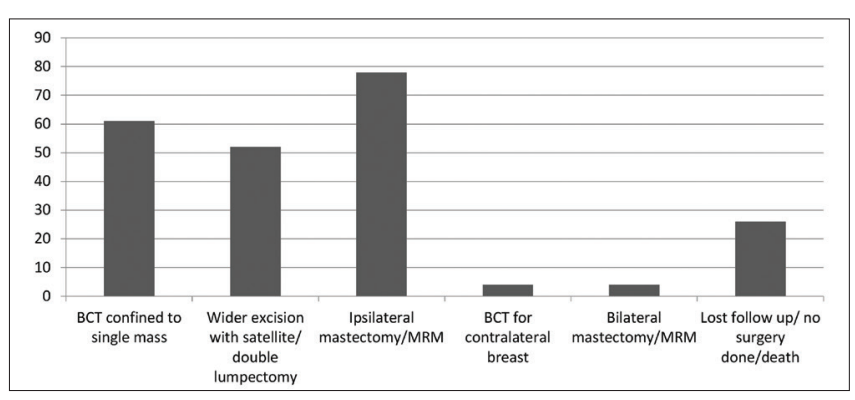

Figure 3. Types of breast surgeries offered to patients with breast cancer

BCT: Breast conservation therapy; MRM: Modified radical mastectomy in situ. Four patients received BCT for malignant satellite masses in the contralateral breast. Bilateral modified radical mastectomy/ mastectomy was performed in four patients with bilateral MF/MC disease. In patients who were lost to follow-up and did not undergo surgery, widespread metastatic disease and death were considered as one category. This category included 26 patients.

\section{Discussion and Conclusion}

Breast cancer is the most common cancer among women, afflicting 2.1 million women each year, and is responsible for the greatest number of cancer-related deaths among women (up to 15\%) (7). Unfortunately, Pakistan has the highest incidence rate of breast cancer in Asia, with an estimated incidence of 83,000 new cases per year (8, 9). Approximately, one of nine women is expected to experience breast cancer at any point in her life (10). Pathologists define synchronous tumors as the presence of two or more foci of cancer with normal breast tissue in between (11). In radiology, cancers are called MF when there is more than one discrete synchronous tumor within the same quadrant of the breast with interlesional distance of $\leq 5 \mathrm{~cm}$ and $\mathrm{MC}$ when multiple cancers are located in different quadrants of the breast and the distance between them is $>5 \mathrm{~cm}(1,12)$.

The estimated prevalence rate of MMBC ranges from $4 \%$ to $65 \%$ of all breast carcinomas; this variability is mainly due to differences in definitions used, lack of standardization in the gross examination, and variability in the extent of sampling of breast specimens (13-15). Moreover, the annual incidence of unsuspected synchronous cancer in contralateral breast varies between $0.3 \%$ and $3 \%$. This variation is attributed to different definitions of synchronicity $(16,17)$.

USG is a useful technique in the evaluation of breast lump, as it is inexpensive and easily available technique that precisely characterizes satellite masses and guides diagnostic interventional procedures.

In the BI-RADS classification established by ACR, breast masses are classified as category 2 (benign), category 3 (likely benign), category 4 a-c (suspicious for malignancy), and category 5 (highly suggestive of malignancy). This classification is based on the following sonographic features: shape, margin, orientation, echo pattern, and posterior features (6). Sonographic features that suggest benign lesion are as follows: circumscribed margins, oval shape, parallel orientation, hyperechoic/isoechoic or mildly hypoechoic, posterior acoustic enhancement, and absence of any malignant features (18, 19). Malignant masses are hypoechoic with irregular/indistinct/ spiculated margins, without parallel orientation and posterior acoustic shadowing $(18,20)$. Color Doppler USG is often considered of limited value because of the significant overlap between vascularization of malignant and benign masses. Nevertheless, in certain situations, it does help resolve the issue, particularly when there is significant vascularity in highly cellular tumors (21-23). In the landmark study in 1995, Stavros et al. (18) classified solid breast masses as benign, indeterminate, or malignant as sonographic features, compared such features with biopsy results, and concluded that sonography can be used to accurately classify some solid masses as benign (99.5\% NPV; $98.4 \%$ sensitivity), allowing imaging follow-up, instead of biopsy. Kwak et al. (24) characterized breast masses according to BI-RADS US criteria and found no statistically significant difference for sensitivity and NPV between FNA cytology and USG.

However, various benign diseases appear as irregular hypoechoic masses and mimic malignancy. Kim et al. (25) classified such lesions into four 
groups, namely, iatrogenic or trauma-related breast lesions (foreign body reaction, fat necrosis, or fibrotic scar), inflammations (abscess, idiopathic granulomatous lobular mastitis, or diabetic mastopathy), proliferative diseases (sclerosing adenosis, apocrine metaplasia, or fibrocystic change), and benign breast tumors (intraductal papilloma, fibroadenoma, or tubular adenoma). Moon et al. (26) evaluated the efficacy of USG in the detection of MF, MC, and contralateral cancers and its effects on therapeutic decisions, and they found a sensitivity of $100 \%$, specificity of $51 \%$, PPV of $64 \%$, NPV of $100 \%$, and therapy change in $32(16 \%)$ patients.

In our study, comparison of the final pathological diagnosis with sonographic classification into benign and malignant classification had different statistical outcome as compared with that reported in aforementioned studies. One possible explanation is that our population consisted of patients with breast cancer alone, and synchronous satellite masses were assessed rather than the primary presenting breast mass; therefore, results can be different from the screened population.

The diagnosis of MF/MC greatly influences the management plan, particularly the choice of surgery; therefore, complete radiological workup and pathological evaluation of synchronous satellite masses is mandatory. In our institution, clinical presentation, risk factors, past medical and surgical history, radiological investigations (size, morphology and number of masses, interlesional distance, axillary lymph node status, and metastatic workup), pathology reports (histopathology/FNA cytology of each mass/lymph node and receptor status), and nuclear medicine bone scan of all patients with breast cancer are discussed in MDT meeting, which comprises consultants from breast surgery, radiology, nuclear medicine, pathology, medical oncology, and radiotherapy departments. Then, the management plan, including the type of surgery, is decided. Patients with a single invasive tumor are usually indicated for lumpectomy. Those with more than one invasive tumor can be selected for BCT (wider excision including the satellite mass if it is located closely or wireguided excision of the satellite mass if it is located at a greater distance or located in the opposite quadrant) or mastectomy depending on the size and number of invasive tumors and patient's breast size, risk factors, post-chemotherapy response, and tumor biology. If neoadjuvant chemotherapy followed by BCT is considered for a patient with $\mathrm{MC} / \mathrm{MF}$ disease, metallic clips are placed under image guidance in biopsy-proven malignant masses before neoadjuvant chemotherapy and US-guided wire placement, followed by postwire mammogram, are performed for preoperative localization for surgery (Figure 4). In cases of upfront surgery for MC/MF disease, US-guided wire localization is planned in MDT for non-palpable mass/masses (Figure 5).

As benign masses can have malignant sonographic appearance and increased risk of malignancy in benign-appearing masses in patients with breast cancer, we strongly believe in obtaining US-guided biopsies for satellite masses (27). This prevents unnecessary surgical excision for benign masses, and US follow-up can be performed instead. Similarly, pathological evidence of $\mathrm{MF} / \mathrm{MC}$ changes the surgical management of patients with breast cancer (Figure 6).

This study had several limitations. First, US-guided FNA had been performed for some patients, so the exact pathological diagnosis was not available; instead, a broader diagnosis of mammary carcinoma color Doppler was not assessed in a significant number of satellite masses (227 masses; 78.8\%). Third, interobserver variability in the interpretations of sonograms among the readers was not evaluated. Fourth, this study has a retrospective single-center design; however, our hospital is the leading cancer hospital and provides services to
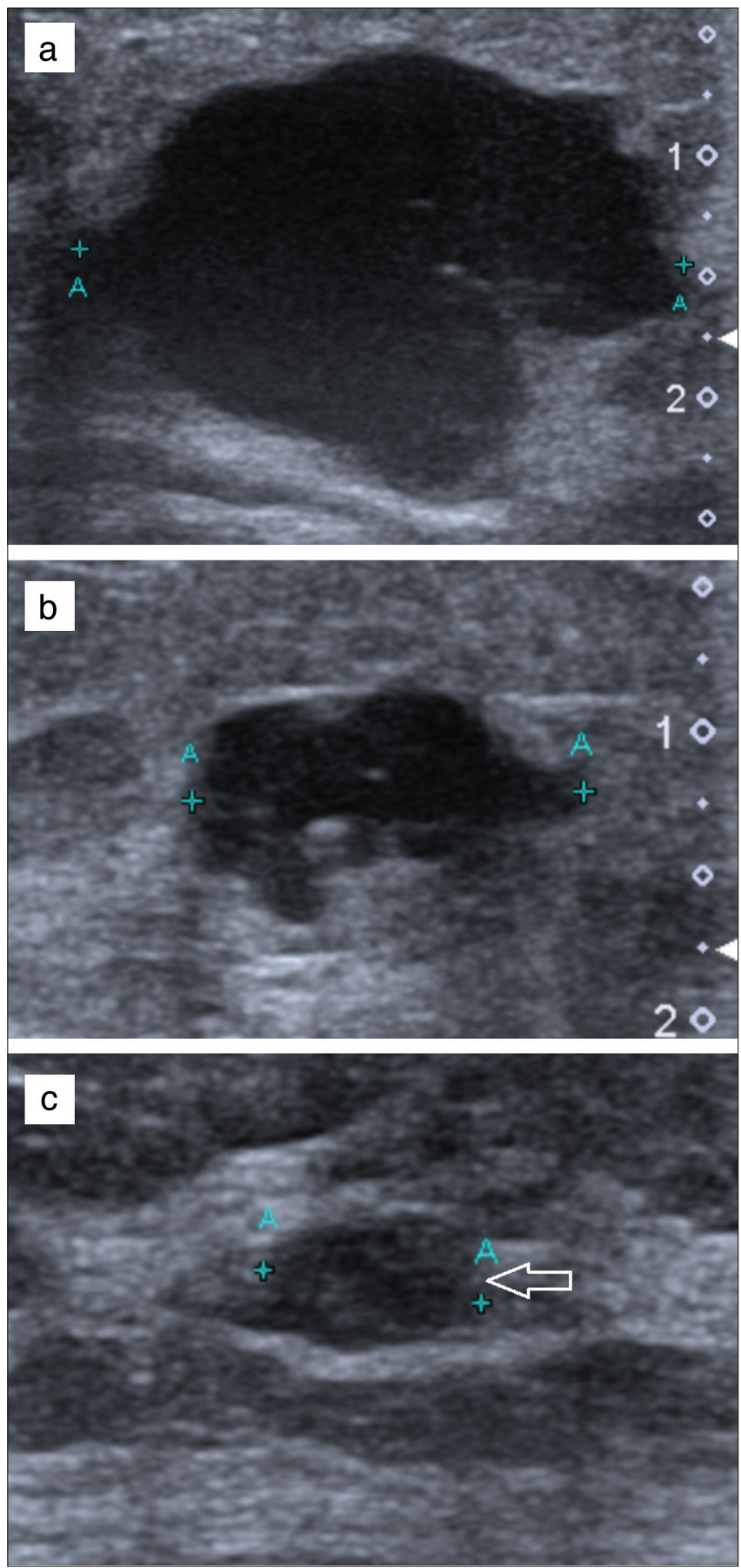

Figure 4. A 65-year-old woman with invasive ductal carcinoma (IDC) grade III in the upper inner quadrant of the right breast (1 o'clock) (a) A small hypoechoic satellite mass with irregular margins and parallel orientation at 1 o'clock (b) and a circumscribed hypoechoic satellite mass with parallel orientation at 10 o'clock (arrow) (c) were sonographically classified as malignant and benign, respectively. Histopathology showed IDC grade III with medullary features and benign breast parenchyma with sclerosing adenosis, respectively. Clips were placed in the 1 o'clock main mass and satellite mass, and the patient underwent right breast double-wire localization lumpectomy after neoadjuvant chemotherapy 

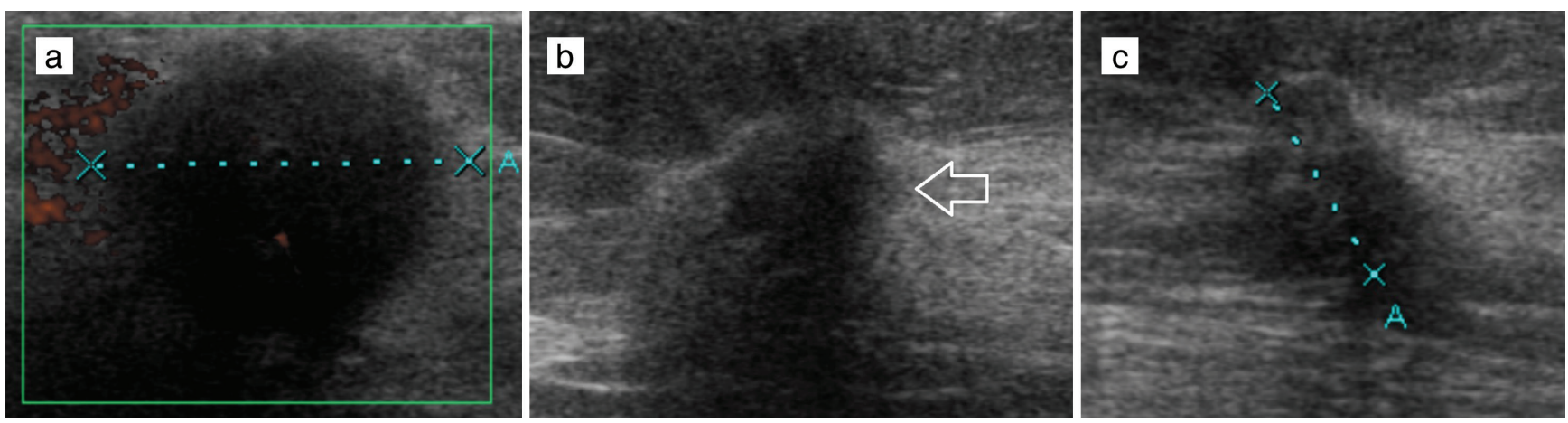

Figure 5. A 62-year-old woman with invasive ductal carcinoma (IDC) grade II in the right breast (5 o'clock) (a). A small hypoechoic satellite mass with irregular margins and non-parallel orientation in the right breast (10 o'clock) (b) and a hypoechoic satellite mass with irregular margins and non-parallel orientation in the left breast (10 o'clock) (c) were both sonographically classified as malignant; histopathology showed IDC grade I and grade II, respectively, with associated lobular carcinoma in situ. The patient underwent wide local excision for the 5 o'clock right breast mass and wire-localized excision for the two satellite masses along with sentinel lymph node biopsy. This was followed by adjuvant chemotherapy

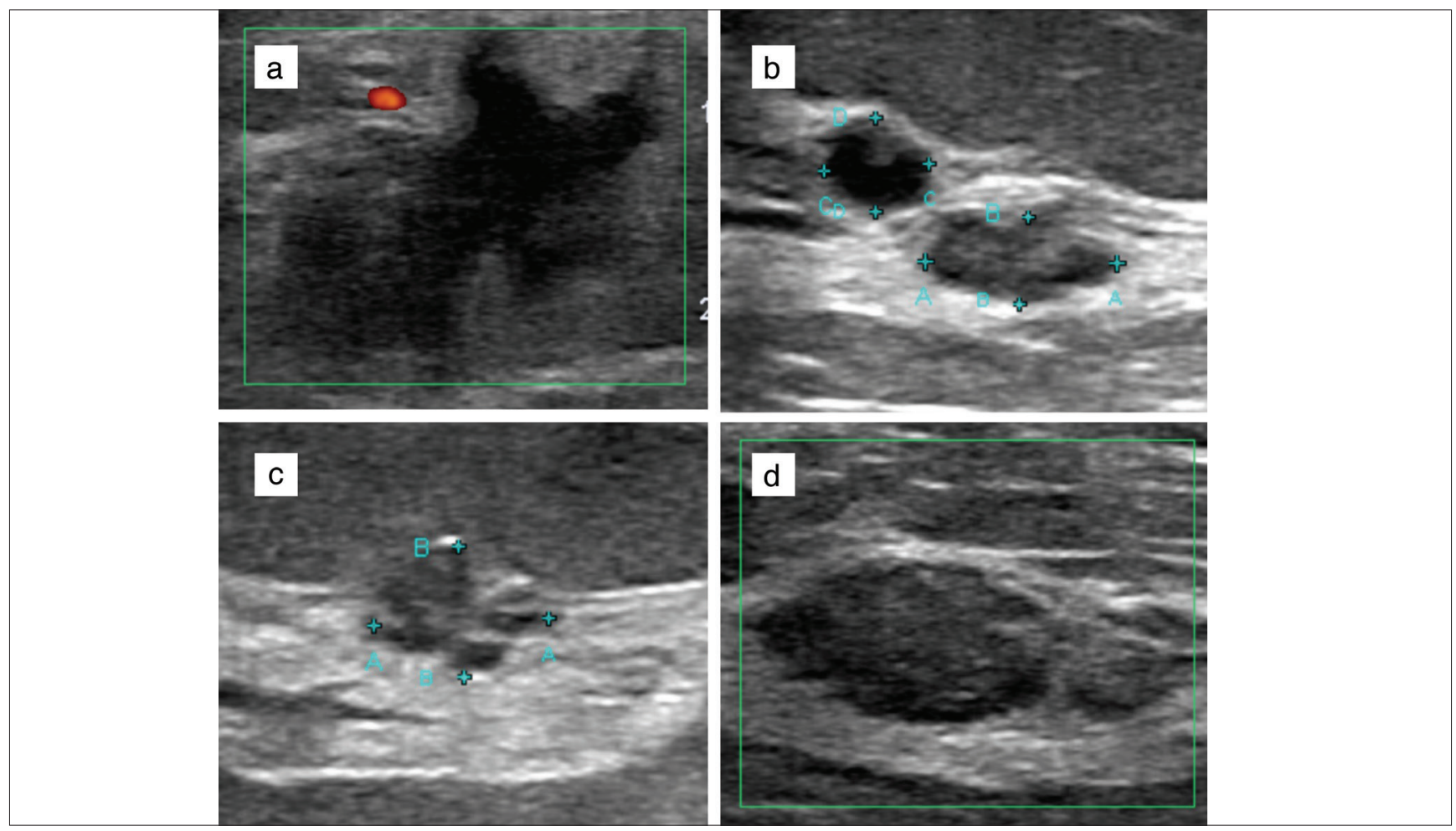

Figure 6. A 47-year-old woman with invasive ductal carcinoma grade III in the left breast (2 o'clock) (a). Two hypoechoic satellite masses with circumscribed margins and parallel orientation in the left breast (3 o'clock) (b) were sonographically classified as benign. A hypoechoic satellite mass with irregular margins and parallel orientation (c) and a hypoechoic mass with microlobulated margins and parallel orientation without increased vascularity on color Doppler (d) at the right breast (6 and 9 o'clock), respectively, were sonographically classified as malignant. Histopathology showed ductal carcinoma in situ cribriform-type intermediate nuclear grade (b) and sclerosing adenosis with usual ductal hyperplasia and stromal fibrosis (c, d). The multidisciplinary team decision was to place clips in the left breast 2 o'clock main mass and 3 o'clock satellite, and she underwent left breast double-wire localization lumpectomy post neoadjuvant chemotherapy

patients from the whole country as well as from neighboring countries; therefore, our patient population can be considered diverse. To the best of our knowledge, there had not been any similar study carried out in our part of world.

In conclusion, the number, location, margins, and shape/orientation of synchronous satellite masses in patients with breast cancer can be correctly evaluated by breast USG. US-guided biopsy should be performed to ascertain the histopathological diagnosis. These findings will facilitate the most appropriate management plan. We hope to do prospective studies in future in evaluation of multiple breast masses with combination of mammogram, ultrasound and MRI features.

Acknowledgement: Dr. Farhana Badar (MBBS, MPH) Sr. Biostatistician \& Cancer Epidemiologist Cancer Registry \& Clinical Data Management, Shaukat Khanum Memorial Cancer Hospital \& Research Center. 
Ethics Committee Approval: Our hospital institutional review board approved this retrospective data collection and analysis (no: EX-15-02-19-01, date: 18.03.2019).

Informed Consent: Retrospective study.

Peer-review: Externally-peer reviewed.

\section{Authorship Contributions}

Surgical and Medical Practices: B.R.; Concept: S.R., I.K.N.; Design: S.R., I.K.N., M.A.N.; Data Collection or Processing: S.R., A.J., B.R.; Analysis or Interpretation: S.R.; Literature Search: S.R.; Writing: S.R.; Supervision and Critical Review: I.K.N., M.A.N.

Conflict of Interest: No conflict of interest was declared by the authors.

Financial Disclosure: The authors declared that this study received no financial support.

\section{References}

1. Middleton LP, Vlastos G, Mirza NQ, Eva Singletary S, Sahin AA. Multicentric mammary carcinoma: evidence of monoclonal proliferation. Cancer 2002; 94: 1910-1916. (PMID: 11932891) [Crossref]

2. Kim SJ, Ko EY, Shin JH, Kang SS, Mun SH, Han BK, et al Application of sonographic BI-RADS to synchronous breast nodules detected in patients with breast cancer. AJR Am J Roentgenol 2008; 191: 653-658. (PMID: 18716090) [Crossref]

3. Atkins H, Hayward JL, Klugman DJ, Wayte AB. Treatment of early breast cancer: a report after ten years of a clinical trial. Br Med J 1972; 2: 423429. (PMID: 4624222) [Crossref]

4. Fisher B, Bauer M, Margolese R, Poisson R, Pilch Y, Redmond C, et al. Five-year results of a randomized clinical trial comparing total mastectomy and segmental mastectomy with or without radiation in the treatment of breast cancer. N Engl J Med 1985; 312: 665-673. (PMID: 3883167) [Crossref]

5. Veronesi U, Cascinelli N, Mariani L, Greco M, Saccozzi R, Luini A, et al. Twenty-year follow-up of a randomized study comparing breastconserving surgery with radical mastectomy for early breast cancer. N Engl J Med 2002; 347: 1227-1232. (PMID: 12393819) [Crossref]

6. Mendelson EB, Böhm-Vélez M, Berg WA, Whitman GJ, Feldman MI, Madjar H. ACR BI-RADS ultrasound. ACR BI-RADS Atlas, Breast Imaging Reporting and Data System. $5^{\text {th }}$ ed. Reston, VA: American College of Radiology; 2013: 1-73.

7. WHO | Breast cancer. (serial online). Last Accessed Date: 21.01.2020. Available from: https:/www.who.int/cancer/prevention/diagnosisscreening/breast-cancer/en/

8. Breast Cancer growing at alarming rate in Pakistan. The Nation. (serial online) 2010 March 19. Last Accessed Date: 21.01.2020. Available from: https://nation.com.pk/19-Mar-2019/breast-cancer-growing-at-alarmingrate-in-pakistan

9. Pakistan has highest breast cancer rate in Asia: experts. Daily Times. (serial online) 2017 October 27. Available from: https://dailytimes. com.pk/131023/pakistan-highest-breast-cancer-rate-asia-experts (Last Accessed Date: 21.01.2020)

10. Sohail S, Alam SN. Breast cancer in pakistan - awareness and early detection. J Coll Physicians Surg Pak 2007; 17: 711-712. (PMID: 18182132) [Crossref]

11. Elston CW, Ellis IO. The Breast, $3^{\text {rd }}$ ed. Churchill Livingstone; 1998: 386-390. [Crossref]
12. Sardanelli F, Giuseppetti GM, Panizza P, Bazzocchi M, Fausto A, Simonetti G, et al. Sensitivity of MRI versus mammography for detecting foci of multifocal, multicentric breast cancer in fatty and dense breasts using the whole-breast pathologic examination as a gold standard. AJR Am J Roentgenol 2004; 183: 1149-1157. (PMID: 15385322) [Crossref]

13. Coombs NJ, Boyages J. Multifocal and multicentric breast cancer: does each focus matter? J Clin Oncol 2005; 23: 7497-7502. (PMID: 16234516) [Crossref]

14. Holland R, Veling SH, Mravunac M, Hendriks JH. Histologic multifocality of tis, T1-2 breast carcinomas implications for clinical trials of breast-conserving surgery. Cancer 1985; 56: 979-990. (PMID: 2990668) [Crossref]

15. Bendifallah S, Werkoff G, Borie-Moutafoff C, Antoine M, Chopier J, Gligorov J, et al. Multiple synchronous (multifocal and multicentric) breast cancer: clinical implications. Surg Oncol 2010; 19: 115-123. (PMID: 20615686) [Crossref]

16. Hartman M, Hall P, Edgren G, Reilly M, Lindstrom L, Lichtenstein P, et al. Breast cancer onset in twins and women with bilateral disease. J Clin Oncol 2008; 26: 4086-4091. (PMID: 18591548) [Crossref]

17. McCaul KA, Anthony K. Bilateral breast cancer incidence and survival. [PhD thesis]. North Terrace, ADELAIDE SA 5005: University of Adelaide; 2006. Available from: University of Adelaide, School of Population Health and Clinical Practice, Library E-Reserve Available at: https://digital.library.adelaide.edu.au/dspace/handle/2440/37870

18. Stavros AT, Thickman D, Rapp CL, Dennis MA, Parker SH, Sisney GA. Solid breast nodules: use of sonography to distinguish between benign and malignant lesions. Radiology 1995; 196: 123-134. (PMID: 7784555) [Crossref]

19. Mainiero MB, Goldkamp A, Lazarus E, Livingston L, Koelliker SL, Schepps B, et al. Characterization of breast masses with sonography: can biopsy of some solid masses be deferred? J Ultrasound Med 2005; 24: 161-167. (PMID: 15661946) [Crossref]

20. Gokhale S. Ultrasound characterization of breast masses. Indian J Radiol Imaging 2009; 19: 242. (PMID: 19881096) [Crossref]

21. Svensson WE, Pandian AJ, Hashimoto H. The use of breast ultrasound color Doppler vascular pattern morphology improves diagnostic sensitivity with minimal change in specificity. Ultraschall Med 2010; 31: 466-474. (PMID: 20094978) [Crossref]

22. Lee WJ, Chu JS, Huang CS, Chang MF, Chang KJ, Chen KM. Breast cancer vascularity: color Doppler sonography and histopathology study. Breast Cancer Res Treat 1996; 37: 291-298. (PMID: 8825140) [Crossref]

23. Tozaki M, Fukuma E. Does power Doppler ultrasonography improve the BI-RADS category assessment and diagnostic accuracy of solid breast lesions? Acta Radiol 2011; 52: 706-710. (PMID: 21596798) [Crossref]

24. Kwak JY, Kim EK, Park HL, Kim JY, Oh KK. Application of the breast imaging reporting and data system final assessment system in sonography of palpable breast lesions and reconsideration of the modified triple test. J Ultrasound Med 2006; 25: 1255-1261. (PMID: 16998097) [Crossref]

25. Kim YR, Kim HS, Kim HW. Are irregular hypoechoic breast masses on ultrasound always malignancies?: a pictorial essay. Korean J Radiol 2015; 16: 1266-1275. (PMID: 26576116) [Crossref]

26. Moon WK, Noh DY, Im JG. Multifocal, multicentric, and contralateral breast cancers: bilateral whole-breast US in the preoperative evaluation of patients. Radiology 2002; 224: 569-576. (PMID: 12147858) [Crossref]

27. Taskin F, Koseoglu K, Ozbas S, Erkus M, Karaman C. Sonographic features of histopathologically benign solid breast lesions that have been classified as BI-RADS 4 on sonography. J Clin Ultrasound 2012; 40: 261265. (PMID: 22508447) [Crossref] 
Rehman et al. Sonography in Breast Cancer Satellite Masses

Appendix 1. Sonographic features

Benign features

1. Margins

2. Shape/orientation

3. Echogenicity

4. Vascularity on color Doppler

Absent
Circumscribed

Oval, parallel

Hyperechoic, isoechoic, or hypoechoic

\section{Malignant features}

Irregular, spiculated, and ill-defined microlobulated

Not parallel

Hypoechoic lesions

Increased 